\title{
Review
}

\section{Measurement of factor XIII activity in plasma}

\author{
Éva Katona ${ }^{1}$, Krisztina Pénzes ${ }^{1}$, Éva Molnár ${ }^{1}$ \\ and László Muszbek ${ }^{1,2, *}$ \\ ${ }^{1}$ Clinical Research Center, University of Debrecen, Medical \\ and Health Science Center, Debrecen, Hungary \\ ${ }^{2}$ Thrombosis, Haemostasis and Vascular Biology Research \\ Group of the Hungarian Academy of Sciences, University \\ of Debrecen, Medical and Health Science Center, Debrecen, \\ Hungary
}

\begin{abstract}
Coagulation factor XIII (FXIII) is converted by thrombin and $\mathrm{Ca}^{2+}$ into an active transglutaminase (FXIIIa) in the final phase of coagulation cascade. Its main function is the mechanical stabilization of fibrin clot and its protection from fibrinolysis by cross-linking of fibrin chains and $\alpha_{2}$-plasmin inhibitor to fibrin. In non-substituted patients FXIII deficiency is a severe hemorrhagic diathesis, not infrequently with fatal consequences. The main reason for using FXIII assays is the diagnosis of FXIII deficiency. The aim of this review is to provide a comprehensive critical evaluation of the methods reported for the determination of FXIII activity in the plasma. Such methods are based on two principles: 1) measurement of labeled amines incorporated by FXIIIa into a glutamine residue of a substrate protein, 2) monitoring ammonia released from a peptide bound glutamine residue by FXIIIa using NAD(P)H dependent glutamate dehydrogenase indicator reaction. The incorporation assays are sensitive, but cumbersome and time-consuming, they are difficult to standardize and cannot be automated. The ammonia release assays are less sensitive, but quick, well standardized, and can be automated; this type of assay is recommended for the screening of FXIII deficiency. The traditional clot solubility assay should not be used for this purpose.
\end{abstract}

Keywords: factor XIII; factor XIII assays; factor XIII deficiency.

*Corresponding author: László Muszbek, MD, PhD, Clinical Research Center, University of Debrecen, Medical and Health Science Center, 98, Nagyerdei krt., Debrecen, 4032, Hungary Phone: +36 52 431956, Fax: +36 52340011 ,

E-mail: muszbek@med.unideb.hu

Received October 7, 2011; accepted January 23, 2012;

previously published online February 23, 2012

\section{Introduction}

Coagulation factor XIII (FXIII) is a zymogen of tetrameric structure (FXIII- $\mathrm{A}_{2} \mathrm{~B}_{2}$ ) that consists of two catalytic A subunits (FXIII-A) and two carrier/inhibitory B subunits (FXIII-B). FXIII-A is synthesized by cells of bone marrow origin, while FXIII-B is produced by hepatocytes. The two subunits form complex in the plasma. FXIII-B is in excess and approximately $50 \%$ of it exists in free non-complexed form. FXIII circulates in association with fibrinogen. FXIII is also present in platelets and monocytes/macrophages, however this cellular form is a dimer of FXIII-A (FXIII- $A_{2}$ ), which lacks FXIII-B. In plasma FXIII is activated by the concerted action of thrombin and $\mathrm{Ca}^{2+}$, first thrombin cleaves off the activation peptide of 37 amino acids from the $\mathrm{N}$-terminal end of FXIII-A, then in the presence of $\mathrm{Ca}^{2+}$ FXIII-A and FXIII-B dissociate and the cleaved FXIII- $\mathrm{A}_{2}$ assumes an enzymatically active configuration. The presence of polymerized fibrin highly ( 80 to 100 -fold) accelerates the activation process, and, if fibrin is present, the activation occurs on the surface of fibrin polymers. In cells, in the absence of FXIII-B, the elevation of $\mathrm{Ca}^{2+}$ concentration is sufficient to bring about the active configuration. For more information on the structure and function of FXIII interested readers should consult most recent extensive reviews $(1,2)$.

Activated FXIII (FXIIIa) is a transglutaminase (proteinglutamine: amine $\gamma$-glutamyltransferase, EC 2.3.2.13) that catalyzes an acyl transfer reaction, in which the carboxamide group of a peptide/protein-bound glutamine residue is the acyl donor and an appropriate primary amine substrate is the acyl acceptor $(3,4)$. Figure 1 demonstrates a simplified scheme of the transglutaminase reaction. The reaction proceeds through an oxyanion intermediate (not shown on the Figure), and ammonia is released as the acyl-enzyme intermediate, a thioesther between the peptide bound glutamine and the -SH group of the active-site cysteine of FXIIIa, is formed. Then, in the presence of a substrate primary amine, the acyl group is transferred to the acyl acceptor amine through a second oxyanion intermediate and the amine becomes covalently linked to the $\gamma$-glutamyl residue via a peptide ("isopeptide") bond, and the active-site cysteine becomes deacylated. If the primary amine is the $\varepsilon$-amino group of a peptide/protein-bound lysine residue, the end-result is the cross-linking of two peptide chains. The measurement of released ammonia, (labeled) amine incorporated into a protein and the cross-linked product (see in red ellipses on Figure 1) all have been utilized for the measurement of FXIII activity. 


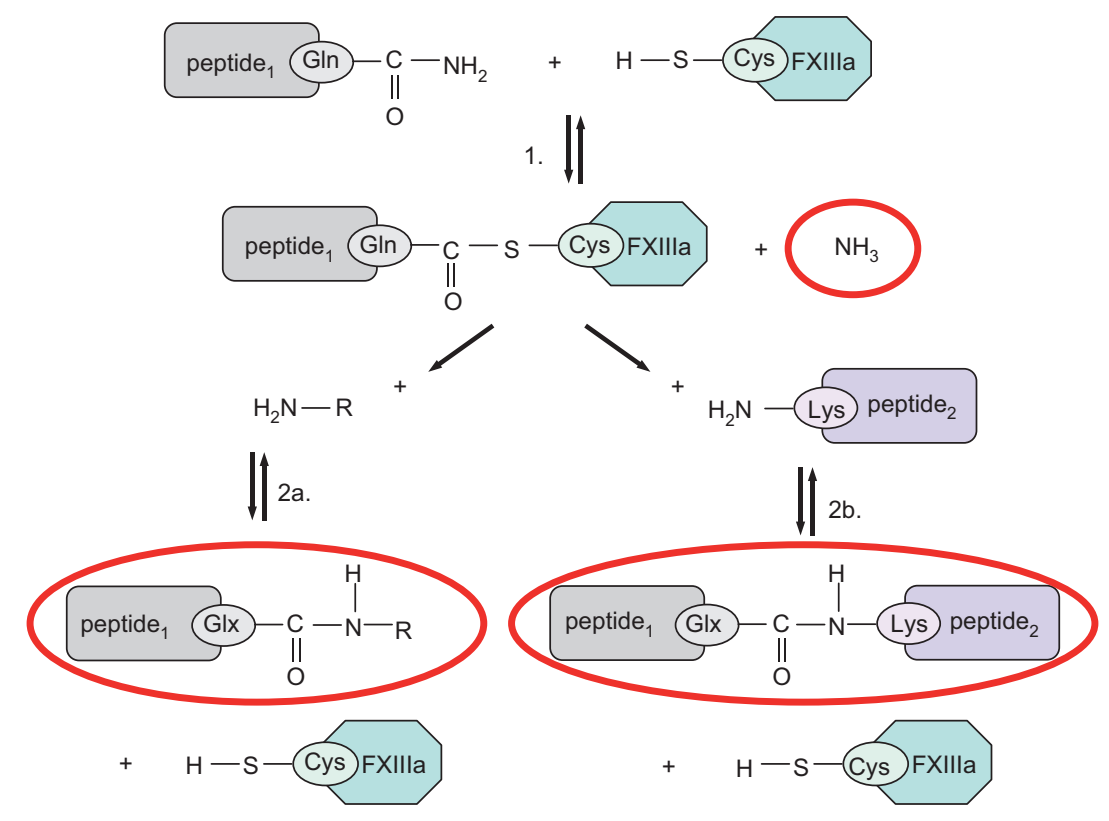

Figure 1 Schematic representation of the transglutaminase reaction catalyzed by activated FXIII (FXIIIa). Different FXIII assays are based on the determination of the products encircled by red ellipses.

The main physiological function of plasma FXIII is the cross-linking of fibrin $\gamma$-chains into dimers and $\alpha$-chains into high molecular mass polymers. In addition it cross-links $\alpha_{2}-$ plasmin inhibitor, the main physiological inhibitor of plasmin, to fibrin $\alpha$-chain; first a hetero-tetramer is formed, then, it becomes incorporated into $\alpha$-chain polymers. This way the action of FXIIIa makes the fibrin network resistant to shear stress and protects it from prompt elimination by the powerful fibrinolytic enzyme, plasmin (2).

There are two reasons why FXIII is determined in clinical practice and clinical research: 1) the diagnosis of FXIII deficiencies and monitoring FXIII substitution of deficient patients; 2) elevated FXIII level might represent a risk factor of thrombotic diseases in certain conditions. The latter is a topic of clinical research rather then routine diagnostics (5).

\section{Factor XIII deficiencies}

Inherited FXIII deficiencies are classified as FXIII-A and FXIII-B deficiency. The former is a rare bleeding disorder, one diagnosed case in a population of two million. Its frequency might be ten times higher in populations in which consanguineous marriage is a common practice. It can be classified as type I deficiency, a quantitative defect due to the decreased synthesis of the protein, and type II deficiency, which is characterized by normal or near normal concentration of FXIII-A with a functional defect. It is a very severe bleeding diathesis in the majority of patients not being on appropriate prophylactic treatment. Umbilical stump bleeding appears in $80 \%$ of FXIII-A deficient newborns, later in life intramuscular, subcutaneous, and unfortunately, also intracranial hemorrhage occur in a high percentage of patients with serious, occasionally fatal, outcome. For this reason, prophylactic FXIII supplementation is mandatory in severe FXIII-A deficiency. FXIII-A deficiency is frequently associated with impaired wound healing and leads to abortion in pregnant women. Repeated spontaneous abortions, even if the bleeding symptoms are only moderate, indicate investigations for FXIII-A deficiency. Six families with inherited FXIII-B deficiency have also been reported (6). In these cases the clinical symptoms were milder.

Acquired deficiency due to a neutralizing or non-neutralizing autoantibody against either of the FXIII subunits also results in severe, often life-threatening bleeding symptoms. It may accompany an autoimmune disease, most frequently systemic lupus erythematosus, or may also occur in the absence of any disease in elderly patients. Less severe acquired FXIII deficiencies may be due to consumption or decreased synthesis of FXIII subunits. In these cases the extent of decrease in plasma FXIII activity is moderate, rarely goes below $20 \%$. Most recent reviews provide detailed information on the clinical symptoms in FXIII deficiencies and on the classification of the disease (7-9).

The official communication of the International Society on Thrombosis and Haemostasis, Scientific, and Standardization Committee (ISTH, SSC) recommends the following algorithm for the diagnosis and classification of FXIII deficiencies (7):

(i) A quantitative plasma FXIII activity assay is to be used as "first line" screening test (see later).

(ii) If it is decreased, the subtype of FXIII deficiency is established by the following tests.

a) Measurement of complex FXIII (FXIII- $\mathrm{A}_{2} \mathrm{~B}_{2}$ ) antigen concentration in the plasma. If its concentration is decreased, FXIII-A and FXIII-B antigens should also be measured. 
b) Measurement of FXIII activity and FXIII-A antigen in platelet lysate.

(iii) If autoantibody against a FXIII subunit is suspected:

a) Mixing study, for the detection of neutralizing antibodies against FXIII-A (determination of FXIII activity in a mixture of normal plasma and the patient's plasma).

b) The detection of non-neutralizing antibodies in binding assays (the binding of auto-antibodies to purified FXIII$\mathrm{A}_{2}$ or FXIII-B, usually in an ELISA type set-up).

(iv) Supplementary test for the evaluation of fibrin crosslinking [clot solubility test or sodium dodecyl sulfate polyacrylamide gel electrophoresis (SDS PAGE) analysis of washed plasma clot].

(v) Exploration of the molecular genetic defect.

The supporting information to this communication provides further practical details in this matter.

Table 1 indicates the generally reported FXIII values in different subclasses of FXIII deficiencies as it was published in the official ISTHSSC communication (7).

Due to methodological problems (see later) no evidencebased data are available on the correlation between the severity of the disease and plasma FXIII activity. A further difficulty is that FXIII deficiency is one of the most unpredictable conditions among coagulation factor deficiencies; in non-substituted patients life-threatening spontaneous bleeding might follow long non-symptomatic intervals. The values given below are only for orientation; individual patients may behave differently. It is generally accepted that severe bleeding diathesis occurs at FXIII level below 3\% of FXIII activity. Patients with plasma FXIII activity between $5 \%$ and $30 \%$ may bleed in hemostatic stress situations. The target pre-dose value in substituted patients is $>5 \%$ FXIII activity. This is $>10 \%$ during pregnancy and $>30 \%$ during labor. During major surgery FXIII activity $>50 \%$ is considered safe.

\section{The effect of plasma FXIII level on the risk of thrombotic diseases}

Earlier studies on association of the risk of coronary artery disease (CAD) and plasma FXIII levels included a relatively low number of male or predominantly male patients. The critical evaluation of these studies was recently published (5). The results of these studies were inconclusive, which was partly due to methodological problems (interference by FXIII-A Val34Leu polymorphism, see later) and partly to not differentiating between the risk of coronary sclerosis without myocardial infarction and the risk of the acute event, i.e., myocardial infarction developed in atherosclerotic coronary vessels.

In a relatively large study involving 955 patients (377 females and 578 males) undergoing coronary angiography, participants were sub-grouped according to the presence or absence of significant coronary sclerosis and according to the presence or absence of the history of myocardial infarction (10). In females, but not in males, adjusted FXIII levels were moderately, but significantly elevated in patients with the history of myocardial infarction. FXIII levels in the upper tertile represented a 2.5 to 3.0-fold risk of myocardial infarction only in females. Elevated FXIII level did not increase the risk of developing significant coronary sclerosis. Elevated FXIII level also exerted a similar gender-specific effect on the risk of peripheral artery disease (11).

A recent review (12) enlists only two studies $(13,14)$ on the association of FXIII level with the risk of venous thromboembolism. Elevated FXIII-A levels did not influence the risk of venous thromboembolism in a recent nested case-control study involving 462 participants who developed venous thromboembolism and 1047 participants who remained free of it (14). Further studies should explore if there is any gender or age specific difference.

Table 1 Diagnosis and classification of FXIII deficiencies.

\begin{tabular}{|c|c|c|c|c|c|c|}
\hline \multirow[t]{2}{*}{ Deficiency } & \multicolumn{4}{|l|}{ Plasma } & \multicolumn{2}{|l|}{ Platelet } \\
\hline & FXIII activity & FXIII- $\mathrm{A}_{2} \mathrm{~B}_{2}$ antigen & FXIII-A antigen & FXIII-B antigen & FXIII activity & FXIII-A antigen \\
\hline \multicolumn{7}{|l|}{ Inherited } \\
\hline \multicolumn{7}{|l|}{ FXIII-A deficiency } \\
\hline Type I (quantitative) & $\downarrow \downarrow \downarrow$ & $\downarrow \downarrow \downarrow$ & $\downarrow \downarrow \downarrow$ & $>30 \%$ & $\downarrow \downarrow \downarrow$ & $\downarrow \downarrow \downarrow$ \\
\hline Type II (qualitative) & $\downarrow \downarrow \downarrow$ & $\downarrow-n$ & $\downarrow-n$ & $>30 \%$ & $\downarrow \downarrow \downarrow$ & $\downarrow-n$ \\
\hline FXIII-B deficiency & $\downarrow \downarrow$ & $\downarrow \downarrow \downarrow$ & $\downarrow \downarrow$ & $\downarrow \downarrow \downarrow$ & $\mathrm{n}$ & $\mathrm{n}$ \\
\hline \multicolumn{7}{|l|}{$\begin{array}{l}\text { Autoantibody against FXIII } \\
\text { Anti FXIII-A }\end{array}$} \\
\hline Neutralizing & $\downarrow \downarrow \downarrow$ & $\downarrow-n$ & $\downarrow-n$ & $>30 \%$ & $\mathrm{n}$ & $\mathrm{n}$ \\
\hline Non-neutralizing & $\downarrow \downarrow \downarrow$ & $\downarrow \downarrow \downarrow$ & $\downarrow \downarrow \downarrow$ & $>30 \%$ & $\mathrm{n}$ & $\mathrm{n}$ \\
\hline Anti-FXIII-B & $\downarrow \downarrow \downarrow$ & $\downarrow \downarrow \downarrow$ & $\downarrow \downarrow \downarrow$ & $\downarrow \downarrow \downarrow$ & $\mathrm{n}$ & $\mathrm{n}$ \\
\hline Other acquired deficiencies ${ }^{\mathrm{a}}$ & $\downarrow$ & $\downarrow$ & $\downarrow$ & $\downarrow-n$ & na & na \\
\hline
\end{tabular}

$\downarrow \downarrow \downarrow$, Highly decreased activity/concentration usually below $3 \%$, and in the majority of cases between non-detectable 1\%; $\downarrow \downarrow$, considerably decreased activity/concentration usually 5\%-10\%; $\downarrow$, slightly decreased activity usually in the range of 20\%-70\%; n, normal; na, non-applicable. The table indicates the generally reported abnormalities, exceptions may occur. acAuired FXIII deficiencies due to increased consumption or decreased synthesis. The Table is reproduced from reference (7) with permission. 


\section{Functional FXIII assays}

Clinical symptoms may indicate FXIII deficiency, but the diagnosis is based on laboratory methods. The generally used screening tests for coagulopathies, prothrombin time, activated partial thromboplastin time and thrombin time are normal in FXIII deficiency, unless there is another concomitant disorder in the clotting system. For instance, lupus anticoagulant with prolonged activated partial thromboplastin time may occur in SLE patients with autoantibodies against FXIII. Full evaluation of the clotting system should include a special test for the detection of FXIII deficiency. The functional "first line" screening assay is the key point in this respect. Due to traditional improper practice in screening, FXIII deficiency is the most under-diagnosed bleeding diathesis.

In FXIII activity assays, FXIII is converted into an active transglutaminase by thrombin and $\mathrm{Ca}^{2+}$ and, in all, except one assay, the products of transglutaminase activity are measured. Figure 1 demonstrates the three possible products that can be determined (see red ellipses), and, accordingly, the methods for the measurement of FXIII activity are based on three different principles: 1) the detection of fibrin cross-linking activity of FXIIIa (clot solubility tests and SDS PAGE analysis of fibrin cross-linking), 2) the determination of small molecular weight amine substrates covalently cross-linked to a protein substrate by FXIIIa (amine incorporation assays), 3) the measurement of ammonia released in the first step of transglutaminase reaction (ammonia release assays). These assay principles and features of assays based on these principles are summarized in Table 2. There is a single assay in which the isopeptidase activity of FXIIIa is measured. Qualitative/semiquantitative and quantitative functional FXIII assays will be discussed in detail.

\section{Qualitative/semiquantitative FXIII assays based on fibrin cross-linking activity}

Clot solubility assays Traditionally, a plasma clot soluble in concentrated urea solution or in dilute acids was considered as the sign of FXIII deficiency. The clot solubility test goes back to the discovery of FXIII when a plasma protein activity that renders the clot insoluble in the presence of $\mathrm{Ca}^{2+}$ was demonstrated (15-17). In the test used for diagnostic purposes plasma samples are clotted by adding thrombin and/or $\mathrm{Ca}^{2+}$ and the clot is then suspended in $5 \mathrm{M}$ or $8 \mathrm{M}$ urea or in $2 \%$ acetic acid or in 1\%-5\% monochloroacetic acid. The method is not standardized, in different applications of the tests the inducers of clotting (thrombin and/or $\mathrm{CaCl}_{2}$ ) and their concentrations, the time allowed for clotting, the type and concentration of the solvents, the time of the detection of solubility, etc. are all variables which influence the limit of detection of FXIII activity. In different reports the limit of detection varied from $<0.5 \%$ to $5 \%$ FXIII activity (1821 ), i.e., depending on the test conditions, moderate and sometimes even severe FXIII deficiencies cannot by detected by these poorly standardized qualitative tests. For these reasons, using the clot solubility tests a considerable part of FXIII deficiencies remain undiagnosed, therefore its use as

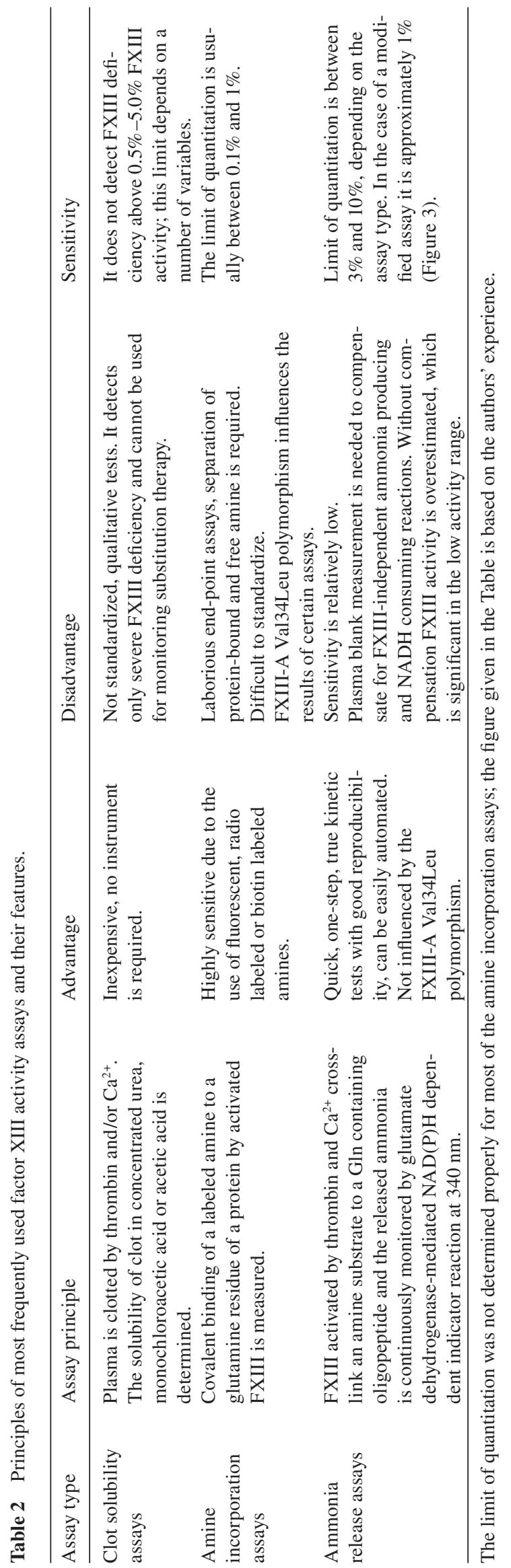

Brought to you by | University of Debrecen (University of Debrecen) 
the screening test for FXIII deficiency was not recommended by the FXIII and Fibrinogen Subcommittee of ISTH, SSC (7). Most recently, based on experiments with mixtures of normal and FXIII deficient plasma, it was claimed that if the clot formed from citrated plasma, i.e., in the absence of added $\mathrm{Ca}^{2+}$, it remains soluble in $2 \%$ acetic acid up-to $18.8 \%$ FXIII activity. This finding has not been confirmed with clinical samples (22).

In summary, it has be emphasized, that, although a positive clot solubility test is strongly indicative of FXIII deficiency, the possibility of deficiency is in no way excluded by a negative test. Another drawback of clot solubility assays are that they cannot be used for monitoring prophylactic treatment and substitution therapy and they are also useless in moderate acquired deficiencies.

Several attempts have been made to design an at least semiquantitative clot solubility assay. Such attempts included the use of FXIII-depleted fibrinogen as "substrate" with different plasma dilutions $(23,24)$ or the modification of clot solubility assay by adding increasing amounts of FXIIIa inhibitors, such as iodoacetate (25) or antiserum specific to FXIII-A subunit (26), to the undiluted plasma sample and determine the amount of inhibitor necessary to neutralize FXIIIa cross-linking activity. In a further modification of the clot lysis assay (27) fibrinogen coated latex beads were exposed to thrombin. The beads aggregated as the consequence of interbead fibrin polymerization and in the absence of FXIII, the aggregates rapidly dissociated in $5 \mathrm{M}$ urea. FXIII dose dependently inhibited the dissociation, which was quantified by turbidimetry. These, in most cases poorly evaluated and standardized assays, did not gain popularity, partly because they are more cumbersome, time consuming and, in some cases, more expensive than commercially available rapid assays.

Fibrin clot analysis by SDS PAGE Fibrin cross-linking in plasma clot can be evaluated by SDS PAGE (28). In this case plasma is clotted by thrombin and $\mathrm{Ca}^{2+}$, then the recovered fibrin clot is extensively washed to remove trapped, noncovalently attached proteins. The washed clot is dissolved in sample buffer and analyzed by SDS PAGE for $\gamma$-chain dimerization and $\alpha$-chain cross-linking. As fibrin $\gamma$-chain dimerization is a highly sensitive function of FXIIIa, even traces (below 1\%) of FXIII activity can be demonstrated by this qualitative or semi-quantitative test. Impaired fibrin crosslinking caused by fibrinogen abnormality can also detected by this test. The procedure is cumbersome and time-consuming and it is only used as a supplementary test in the diagnosis of FXIII deficiency.

\section{Quantitative FXIII assays}

In all quantitative assays, FXIII is first (or during the lag phase of the reaction) activated by thrombin and $\mathrm{Ca}^{2+}$. However, the transformation of fibrinogen into fibrin by thrombin causes difficulties in the separation of free amines from proteinbound amines and fibrin would also interfere with photometric or fluorimetric assays. The elimination of this problem has been solved in the following ways: 1) Traditionally, the denaturation of fibrinogen in plasma samples and detachment of FXIII from fibrinogen prior to the assays was achieved by a short heat treatment at $56^{\circ} \mathrm{C}$ in the presence of glycerol or ethyleneglycol (29). It was expected that such a mild heat treatment separates FXIII from fibrinogen and leaves it intact. However, this was not quite the case and it was shown that a significant unpredictable quantity of FXIII activity was lost during heat treatment (30). A higher recovery of FXIII was achieved when fibrinogen was removed from the plasma by adsorption to bentonite $(30,31)$. Bentonite treatment needed an extra step of centrifugation and now the elimination of fibrinogen by heat treatment and adsorption are only of historical interest; 2 ) The prevention of fibrin polymerization in the FXIII assay mixture by a polymerization inhibitory peptide was first introduced by Miraglia and Greenberg (32) and later it became popular to include peptides, like GPRP or GPRPA, in the FXIII assay mixture (33-35); 3) Finally, in highly sensitive amine incorporation assays the high dilution of plasma samples prevent the formation of fibrin polymers (36-38). It is to be noted that by eliminating fibrinogen and preventing fibrin polymerization, the FXIII activation accelerating effect of polymerized fibrin is lost and rather high thrombin concentration is needed to bring about full activation of FXIII.

Another general problem of FXIII activation concerns one of the polymorphisms of FXIII-A. A Val to Leu amino acid replacement just three amino acids up-stream of the thrombin cleavage site in the activation peptide, turned out to affect significantly the rate of FXIII activation by thrombin. The initial activation of the Leu 34 variant proceeds 2.5 -fold faster, while the activity of fully activated FXIIIa is identical in all genotypes (39-41). Only two studies investigated the influence of FXIII Val34Leu polymorphisms on the measurement of FXIII activity. The assay described by Wilmer et al. measures transglutaminase activity at the initial stage of FXIII activation (36), and the results obtained by this assay reflect the rate of FXIII activation rather than the catalytic concentration. FXIII activity values obtained with the Leu34 variant are significantly higher than those obtained with same concentration of the Val34 variant $(36,42)$. For this reason different expected values were reported for wild type $(39.3 \%-100.4 \%)$, for heterozygote (34.5\%-183.0\%) and for homozygote (96.3\%$226.0 \%$ ) individuals. The assay shows poor correlation with the antigenic assay and with activity assays not influenced by the polymorphism. Users should be aware of this problem. In contrast, the assay described by Kárpáti et al. (34) and very likely also the assay reported by Fickenscher et al. (33), measure the transglutaminase activity of fully activated enzyme and the results are not influenced by FXIII-A Val34Leu genotype. Unfortunately, other assays have not been tested for the effect of FXIII-A Val34Leu polymorphism.

A significant step in the standardization of FXIII assays was the establishment of a WHO reference plasma by the FXIII Working Party of ISTH, SSC (43). This plasma with assigned FXIII activity and plasma FXIII antigen values is available from the National Institute for Biological Standards and Control (Potters bar, UK) and it is to be used for the calibration of reference plasmas by companies producing FXIII assay kits. 
Amine incorporation assays The first quantitative FXIII assays were based on the principle of amine incorporation. Table 3 enumerates the publications on such assays that were used for the determination of FXIII activity in human plasma. In these assays the covalent binding of an acyl acceptor substrate amine to an acyl donor glutamine residue of a protein by FXIIIa is measured. As discussed above the elimination of interfering fibrin formation was fist solved by previous heat treatment of fibrinogen (29, $44,46-49$ ) or by fibrinogen adsorption to bentonite (31). More recently only fibrin polymerization inhibitory peptide $(32,35)$ and prevention of fibrin polymerization by high plasma dilution (35-38) are used for this purpose. One of the burdens of amine incorporation assays is the separation of free non-bound amines from protein linked ones. This was first solved by acid precipitation and removal of unbound amines by repeated washing of the protein precipitate (29, $32,44,45,47,48)$. Later the separation was simplified by the use of substrate proteins covalently or non-covalently bound to a surface $(31,35-38,46)$. The disadvantage of this simplification was that the substrate protein could not be used at saturating concentration, which impaired the precision of the assay.

Casein $(29,38,44-47,49)$, casein derivatives $(31,32,48)$ and fibrinogen (35-37) are the Gln donor substrate proteins used in various amine incorporation assays. Hammerstein casein is a mixture of different casein molecules. In addition to substrate Gln residues they also contain Lys residues, which are substrates for FXIIIa and these Lys residues compete with the amine substrates in the assay. Blocking the Lys residues by methylation or acetylation overcame this problem $(31,32,48,50)$. Besides, there are a number of Gln residues in casein to which FXIIIa cross-links amines with different kinetics. A further problem was the gradual precipitation of substrate casein in the presence of $\mathrm{Ca}$ ions. Acetylated, dephosphorylated $\beta$-casein is exempt of these problems (50), however it is not available commercially and its production is rather laborious. Fibrinogen, as opposed to fibrin, is a rather poor substrate of FXIIIa. However, the adsorption to a surface significantly improves the substrate characteristics of fibrinogen.

Fluorescent dansylcadaverine $(29,49)$, radiolabeled putrescine $(32,44-47)$, or biotinylated cadaverine (biotinamidopentylamine) $(31,35-37)$ are the most frequently used amine substrates in FXIII assays. Putrescine is a diamine and it can provide two primary amine groups for the transglutaminase reaction, i.e., protein-linked putrescine still can offer its remaining free amine for cross-linking two protein molecules. This again, might influence the kinetics of the transglutaminase reaction. Although this is not a problem with other radiolabeled amine substrates, like glycine ethylesther or histamine, these amines have not been used in plasma FXIII assays.

Some of the assays use very long incubation times, up-to $4 \mathrm{~h}$ (Table 3). Activated FXIII is a rather instable enzyme and after 30 min gradually looses its activity at $37^{\circ} \mathrm{C}$ (unpublished observation). For this reason incubation longer than 30 min is not recommended. The measurement of incorporated fluorescent or radiolabeled amines is straightforward. This is not the case with biotinylated cadaverine; in this case a separate detection reaction using streptavidin conjugated with a fluorophore (35) or with an enzyme $(31,36,37)$ is required. The detection using enzymatic (alkaline phosphates or $\beta$-galactosidase) reaction prolongs the assay time and it should be carefully controlled.

In general, the advantage of amine incorporation methods is their high sensitivity due to the use of fluorescent, radiolabeled amines or the detection of the incorporated biotin moieties by an enzymatic reaction. The methods using dansyl-cadaverine can detect as low as $1 \%$ plasma FXIII activity, while the sensitivity of the other methods goes even below that limit. However, the amine incorporation methods are rather troublesome and timeconsuming, to perform an assay takes long hours. They are difficult to standardize and the separation step makes it hardly possible to design a true kinetic assay. A pseudo-kinetic assay can be designed when the incorporation is determined at various times after the initiation of the reaction.

Ammonia release assays The first FXIII assay utilizing the measurement of ammonia released by FXIIIa from its Gln donor protein substrate was published in 1969 (51). In this case ammonia formed in recalcified clotted plasma was estimated. Further early methods determined ammonia formed in plasma or plasma supplemented with fibrinogen by ammonia selective electrode (52) or a modified Berthelot reaction (53). These end-point methods were impractical and required a high volume of plasma, they did not gain popularity.

A new principle was introduced by using glutamate dehydrogenase $(\mathrm{GlDH})$ indicator reaction for monitoring the production of ammonia (30). This principle made it possible to design the first true kinetic FXIII assay (30). In the assay, fibrinogen was removed by bentonite treatment. FXIII was activated by thrombin and $\mathrm{Ca}^{2+}$ during the lag phase of the reaction. FXIIIa cross-linked the amine substrate (ethylamine) to the single substrate Gln residue in acetylated dephosphorylated $\beta$-casein and ammonia was released. The ammonia release was continuously monitored by glutamate dehydrogenase-mediated indicator reaction, in which NADPH became converted into NADP and the decrease in absorbance at $340 \mathrm{~nm}$ was measured.

The above principle was used in the development of two kinetic ammonia release FXIII assays (Figure 2) $(33,34)$. In the new modified assays fibrin formation was prevented by peptides that inhibited fibrin polymerization, the protein substrate was replaced by Gln containing oligopeptides and glycine ethylesther was used as amine substrate (Table 4). Both modified assays are now commercially available as Berichrom FXIII (Siemens, Marburg, Germany), REA-chrom FXIII (Reanal-ker, Budapest, Hungary) and TECHNOCHROM FXIII (Technoclone, Vienna, Austria) tests. These ammonia release assays are quick (only $10 \mathrm{~min}$ ), one-step, true kinetic tests with good reproducibility and can be easily automated. Neither assay is influenced by the FXIII-A Val34Leu polymorphism $(33,34,54)$. They are widely used as screening tests for FXIII deficiency. The Berichrom and TECHNOCHROM assays are compared in Table 4 (the Rea-chrom assay is identical with the TECHNOCHROM assay). The two kits use different Gln containing substrate 


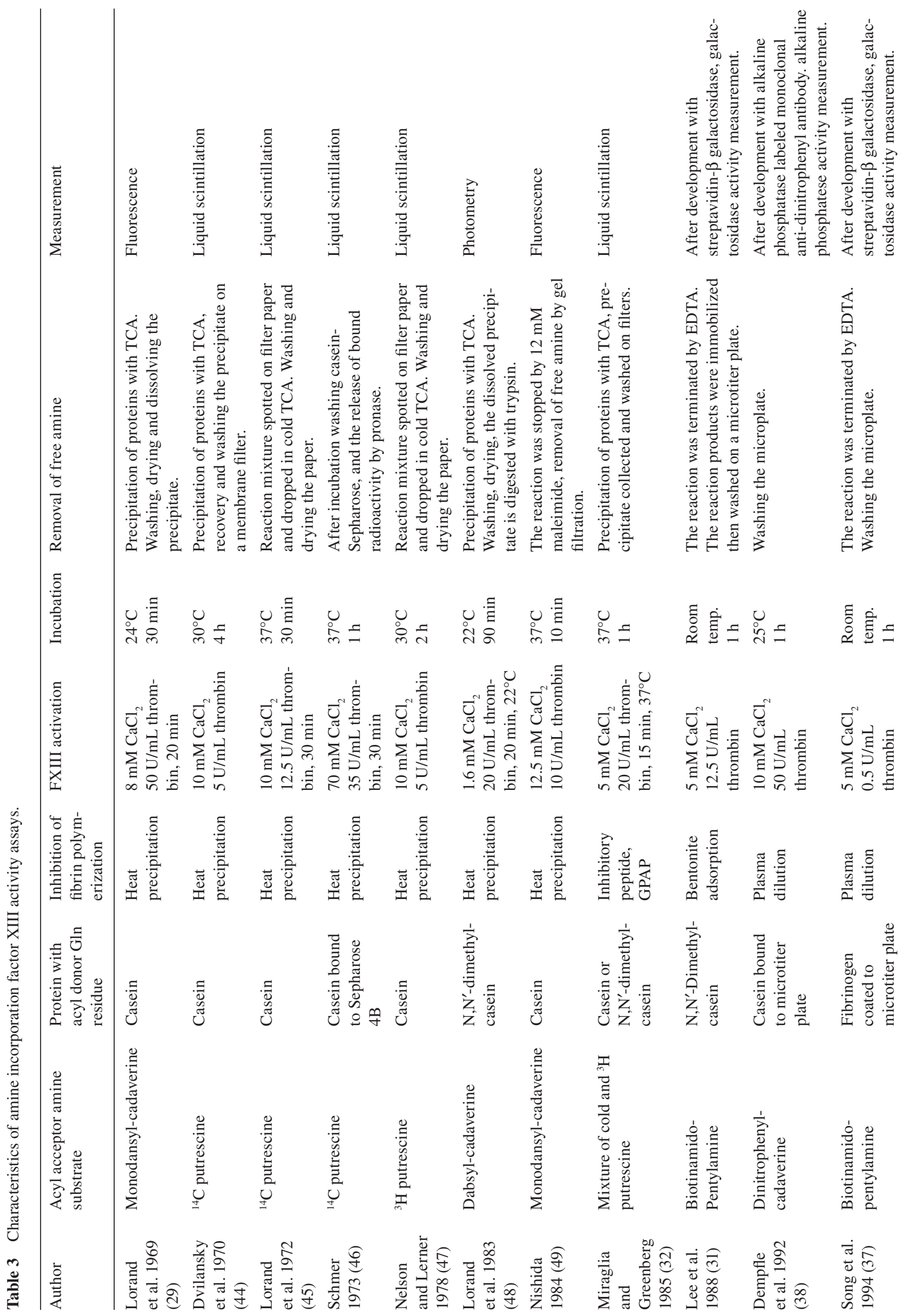


oligopeptides; in the Berichrom assay the decapeptide possesses an amino acid sequence similar to that of $\beta$-casein around the substrate Gln residue, the sequence of the dodecapeptide used in the TECHNOCHROM (and Rea-chrom) kit is identical with the $\mathrm{N}$-terminal sequence of N1-isoform of $\alpha_{2}$-plasmin inhibitor. In the indicator reaction NADH and NADPH are used in the Berichrom and TECHNOCHROM assays, respectively.

One of the problems to be discussed concerning the ammonia release FXIII assays comes from the ammonia producing and NADH consuming reactions in the plasma that result in decreased absorbance at $340 \mathrm{~nm}$ independently of FXIIIa activity. Lactate dehydrogenase present in the plasma is a major source of the NADH consuming side-reaction. The LDH substrate, pyruvate, is also present in the plasma, and its transformation to lactate directly consumes NADH, but not NADPH (55). FXIIIa-independent ammonia production in the plasma samples is another source of non-specific $\mathrm{NAD}(\mathrm{P}) \mathrm{H}$ consumption $(56,57)$. An example for such a reaction is the production of ammonia by the de-amidation of glutamine by $\gamma$-glutamyl transferase present in the plasma. The extent of these side-reactions varies individually and, if not subtracted, results in overestimation of FXIII activity (58). Such an overestimation adds $2 \%-15 \%$ to plasma FXIII activity, which does not seem to cause clinically significant problem around the normal range, however in FXIII deficient patients gives false information on the severity of FXIII deficiency and on the efficiency of replacement therapy ( 9 , $34,54,58)$. The overestimation of FXIII activity in the low activity range by the Berichrom FXIII assay was also demonstrated by surveys of the ECAT external quality assessment program (59). To avoid the overestimation of FXIII activity the subtraction of a plasma blank, in which FXIIIa is inhibited by $1 \mathrm{mM}$ iodoacetamide, is recommended $(34,54,58)$. A reagent for plasma blank measurement is included in the REA-chrom and TECHNOCHROM kits; in the Berichrom assay, plasma blank reagent is to be prepared by the user.

The disadvantage of the above assays is their relatively low sensitivity. In our hands, their limit of quantitation varied 3\%-10\% depending on the instruments used for FXIII activity determination. Unfortunately, this range is important

1. Activation during the lag phase:

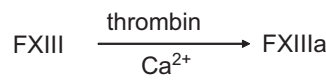

Inhibition of fibrin polymerization by GPRP(A)

2. Transglutaminase reaction:

Gly-OEt + Q-peptide $\stackrel{\text { FXIIla }}{\longrightarrow}$ Q-peptide-Gly-OEt $+\mathrm{NH}_{3}$

3. Monitoring the release of ammonia:

$\mathrm{NH}_{3}+$-ketoglutarate+NAD $(\mathrm{P}) \mathrm{H} \stackrel{\mathrm{GIDH}}{\longrightarrow}$ glutamate + NAD(P)

Figure 2 Kinetic photometric ("chromogenic") factor XIII assays based on monitoring ammonia released during the transglutaminase reaction.

Gly-OEt, glycine ethylesther; Q-peptide, glutamine containing acyl donor substrate peptide; GIDH, glutamate dehydrogenase. 
Table 4 Composition of commercial ammonia release (chromogenic) FXIII activity assays.

\begin{tabular}{lll}
\hline Assay kit & $\begin{array}{l}\text { Berichrom FXIII } \\
\text { Siemens, Marburg, Germany }\end{array}$ & $\begin{array}{l}\text { TECHNOCHROM FXIII } \\
\text { Technoclone, Vienna, Austria }\end{array}$ \\
\hline Fibrin polymerization inhibitory peptide & G-P-R-P-A & G-P-R-P \\
FXIII activator & Thrombin+Ca ${ }^{2+}$ & Thrombin+Ca ${ }^{2+}$ \\
Acyl donor Q-peptide & L-G-P-G-Q-S-K-V-I-G & N-Q-Q-V-S-P-L-T-L-L-L-K \\
Acyl acceptor amine & Glycine ethylesther & Glycine ethylesther \\
Indicator reaction & GlDH with NADH & GlDH with NADPH \\
Plasma blank reagent in the kit & Not included & Included \\
Plasma:reagent volume ratio & $1: 10$ & $1: 10$ \\
\hline
\end{tabular}

The amino acid sequence of the peptide in the Berichrom kit corresponds, with some modification, to the sequence of $\beta$-casein around the substrate Gln residue. The sequence of the dodecapeptide in the TECHNOCHROM kit corresponds to the N-terminal sequence of the FXIIIa substrate $\alpha_{2}$-plasmin inhibitor isoform (N1 $\alpha_{2}$-plasmin inhibitor) (42). GIDH, glutamate dehydrogenase.

for the stratification of disease severity and for controlling the replacement therapy. With a slight modification of the TECHNOCHROM assay, doubling the sample volume and extending the measuring interval after the 5 min lag period from 5 to $10 \mathrm{~min}$, we were able to measure FXIII activity below $5 \%$ with good linearity and acceptable repeatability (Figure 3 ). The modified assay satisfies most of the requirements of clinical diagnostics and monitoring replacement therapy. If an even more sensitive assay is needed, e.g., for studies of phenotypegenotype correlation in the very low activity range, one of the amine incorporation assays is recommended.

Kappel et al. modified the Berichrom FXIII assay (60) by replacing NADH for thio-NADH in the indicator reaction, this way the measuring wavelength was brought into the visible range $(405 \mathrm{~nm})$ commonly available on coagulometers with optical channel. The change of the cofactor however does not eliminate the problem caused by NADH consuming and ammonia producing side reactions (see above) and the modification did not improve the sensitivity of the assay (59).

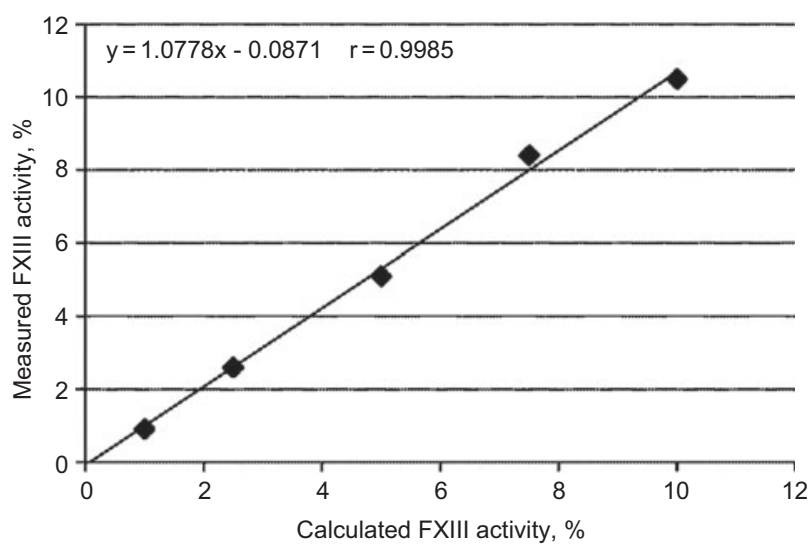

Figure 3 Measurement of FXIII activity in the low activity range by the modified TECHNOCHROM FXIII assay.

A plasma:reagent ratio of 1:5 was used and the measuring interval was $5-15 \mathrm{~min}$ after the initiation of the reaction. The repeatability of the modified test (mean FXIII activity \pm standard deviation) was $7.5 \% \pm 0.36 \%, 1.9 \% \pm 0.3 \%$ and $0.7 \% \pm 0.25 \%$.
FXIII assay based on the determination of isopeptidase activity An interesting concept for the determination of FXIII activity was put forward by Parameswaran et al. (61). It was shown that in certain conditions FXIIIa can exert isopeptidase activity, i.e., as opposed to its cross-linking activity, it can release primary amines bound to a Gln residue in an oligopeptide. They used a primary amine labeled with a quencher, and a peptide substrate that contained a fluorophore. Gradual removal of the quencher containing amine by FXIIIa resulted in increasing fluorescence. This assay was modified and made more sensitive by Oertel et al. (62). They used 2-aminobenzoyl fluorophore labeling at the $\mathrm{N}$-terminus of the peptide corresponding to the $\mathrm{N}$-terminal amino acid sequence of $\alpha_{2}$-plasmin inhibitor and dinitrophenyl cadaverine as internal quencher. Fluorescence was measured at $418 \mathrm{~nm}$ (excitation at $313 \mathrm{~nm}$ ). The reagent is commercially available for research use from Zedira (Darmstadt, Germany). The limit of quantitation (5\% FXIII activity) of the fluorimetric assay does not compare favorably to that of the widely used photometric ammonia release assays. Its correlation with the FXIII-A subunit ELISA was poorer than that of the photometric assay and the correlation with the photometric transglutaminase assay was not very good, either (62). It remains to be seen to what extent the measured isopeptidase activity reflects the physiologically important cross-linking activity of FXIIIa.

\section{Acknowledgments}

The authors were supported by grants from the Hungarian National Research Fund (OTKA-NKTH CNK 80776), from the Hungarian Academy of Sciences (TKI 227) and from the Hungarian Ministry of Health (ETT) The work was also supported by the TÁMOP 4.2.1./B09/1/KONV-2010-0007 and the TÁMOP-4.2.2/B-10/1-2010-0024 projects implemented through the New Hungary Development Plan, co-financed by the European Social Fund.

\section{Conflict of interest statement}

Authors' conflict of interest disclosure: The authors stated that there are no conflicts of interest regarding the publication of this article. Research support played no role in the study design; in the 
collection, analysis, and interpretation of data; in the writing of the report; or in the decision to submit the report for publication.

Research funding: None declared.

Employment or leadership: None declared.

Honorarium: None declared.

\section{References}

1. Komaromi I, Bagoly Z, Muszbek L. Factor XIII: novel structural and functional aspects. J Thromb Haemost 2011;9:9-20.

2. Muszbek L, Bereczky Z, Bagoly Z, Komaromi I, Katona E. Factor XIII: a coagulation factor with multiple plasmatic and cellular functions. Physiol Rev 2011;91:931-72.

3. Iismaa SE, Holman S, Wouters MA, Lorand L, Graham RM, Husain A. Evolutionary specialization of a tryptophan indole group for transition-state stabilization by eukaryotic transglutaminases. Proc Natl Acad Sci USA 2003;100:12636-41.

4. Iismaa SE, Mearns BM,Lorand L, Graham RM. Transglutaminases and disease: lessons from genetically engineered mouse models and inherited disorders. Physiol Rev 2009;89:991-1023.

5. Muszbek L, Bereczky Z, Bagoly Z, Shemirani AH, Katona E. Factor XIII and atherothrombotic diseases. Semin Thromb Hemost 2010;36:18-33.

6. Biswas A, Ivaskevicius V, Seitz R, Thomas A, Oldenburg J. An update of the mutation profile of Factor $13 \mathrm{~A}$ and B genes. Blood Rev 2011;25:193-204.

7. Köhler HP, Ichinose A, Seitz R, Ariens RA, Muszbek L. Diagnosis and classification of factor XIII deficiencies. J Thromb Haemost 2011;9:1404-6.

8. Muszbek L, Bagoly Z, Cairo A, Peyvandi F. Novel aspects of factor XIII deficiency. Curr Opin Hematol 2011;18:366-72.

9. Karimi M, Bereczky Z, Cohan N, Muszbek L. Factor XIII Deficiency. Semin Thromb Hemost 2009;35:426-38.

10. Bereczky Z, Balogh E, Katona E, Czuriga I, Edes I, Muszbek L. Elevated factor XIII level and the risk of myocardial infarction in women. Haematologica 2007;92:287-8.

11. Shemirani AH, Szomjak E, Csiki Z, Katona E, Bereczky Z, Muszbek L. Elevated factor XIII level and the risk of peripheral artery disease. Haematologica 2008;93:1430-2.

12. Bereczky Z, Muszbek L. Factor XIII and venous thromboembolism. Semin Thromb Hemost 2011;37:305-14.

13. Van Hylckama Vlieg A, Komanasin N, Ariens RA, Poort SR, Grant PJ, Bertina RM, et al. Factor XIII Val34Leu polymorphism, factor XIII antigen levels and activity and the risk of deep venous thrombosis. Br J Haematol 2002;119:169-75.

14. Cushman M, O'Meara ES, Folsom AR, Heckbert SR. Coagulation factors IX through XIII and the risk of future venous thrombosis: the longitudinal investigation of thromboembolism etiology. Blood 2009; 114:2878-83.

15. Laki K, Lorand L. On the solubility of fibrin clots. Science 1948;108:280.

16. Lorand L, Jacobsen A. Studies on the polymerization of fibrin; the role of the globulin: fibrin-stabilizing factor. J Biol Chem 1958;230:421-34.

17. Tyler HM. A comparative study of the solvents commonly used to detect fibrin stabilisation. Thromb Diath Haemorrh 1966;16:61-8.

18. Jennings I, Kitchen S, Woods TA, Preston FE. Problems relating to the laboratory diagnosis of factor XIII deficiency: a UK NEQAS study. J Thromb Haemost 2003;1:2603-8.

19. Jakobsen E, Godal HC. Simple, semiquantitative test for partial factor XIII (FSF) deficiency. Scand J Haematol 1974;12:366-8.
20. Francis JL. The detection and measurement of factor XIII activity: a review. Med Lab Sci 1980;37:137-47.

21. Chanarin I. Laboratory haematology: an account of laboratory techniques. Edinburgh; New York: Churchill Livingstone, 1989.

22. Shanbhag S, Shetty S, Kulkarni B, Ghosh K. An improved, semi quantitative clot based assay for factor XIII. Haemophilia 2011;17:718-20.

23. Loewy AG, Dunathan K, Kriel R, Wolfinger HL Jr., Fibrinase I. Purification of substrate and enzyme. $J$ Biol Chem 1961;236:2625-33.

24. Bohn H. Isolation and characterization of the fibrin stabilizing factor from human thrombocytes. Thromb Diath Haemorrh 1970;23:455-68.

25. Sigg P. The monoiodoacetate (MIA) tolerance test, a new quantitative method for the fibrin stabilizing factor (factor 13) assay. Thromb Diath Haemorrh 1966;15:238-51.

26. Bohn H, Haupt H. A quantitative determination of factor 13 with anti-factor 13 serum. Thromb Diath Haemorrh 1968;19:309-15.

27. Retzinger GS, Cook BC, Smith RE, McGinnis MC. Quantitation of plasma factor XIIIa activity using fibrin-coated microscopic latex beads. Anal Biochem 1991;195:18-23.

28. Peyvandi F, Tagliabue L, Menegatti M, Karimi M, Komaromi I, Katona E, et al. Phenotype-genotype characterization of 10 families with severe a subunit factor XIII deficiency. Hum Mutat 2004;23:98.

29. Lorand L, Urayama T, De Kiewiet JW, Nossel HL. Diagnostic and genetic studies on fibrin-stabilizing factor with a new assay based on amine incorporation. J Clin Invest 1969;48:1054-64.

30. Muszbek L, Polgar J, Fesus L. Kinetic determination of blood coagulation Factor XIII in plasma. Clin Chem 1985;31:35-40.

31. Lee KN, Birckbichler PJ, Patterson MK Jr. Colorimetric assay of blood coagulation factor XIII in plasma. Clin Chem 1988;34:906-10.

32. Miraglia CC, Greenberg CS. Measurement of blood coagulation Factor XIIIa formation in plasma containing glycyl-L-prolyl-Larginyl-L-proline. Anal Biochem 1985;144:165-71.

33. Fickenscher K, Aab A, Stuber W. A photometric assay for blood coagulation factor XIII. Thromb Haemost 1991;65:535-40.

34. Kárpáti L, Penke B, Katona E, Balogh I, Vamosi G, Muszbek L. A modified, optimized kinetic photometric assay for the determination of blood coagulation factor XIII activity in plasma. Clin Chem 2000;46:1946-55.

35. Kwon MH, Kong DH, Jung SH, Suh IB, Kim YM, Ha KS. Rapid determination of blood coagulation factor XIII activity using protein arrays for serodiagnosis of human plasma. Anal Chem 2011;83:2317-23.

36. Wilmer M, Rudin K, Kolde H, Poetzsch B, Lenz W, Moessmer $\mathrm{G}$, et al. Evaluation of a sensitive colorimetric FXIII incorporation assay. Effects of FXIII Val34Leu, plasma fibrinogen concentration and congenital FXIII deficiency. Thromb Res 2001;102:81-91.

37. Song YC, Sheng D, Taubenfeld SM, Matsueda GR. A microtiter assay for factor XIII using fibrinogen and biotinylcadaverine as substrates. Anal Biochem 1994;223:88-92.

38. Dempfle CE, Harenberg J, Hochreuter K, Heene DL. Microtiter assay for measurement of factor XIII activity in plasma. J Lab Clin Med 1992;119:522-8.

39. Ariens RA, Philippou H, Nagaswami C, Weisel JW, Lane DA, Grant PJ. The factor XIII V34L polymorphism accelerates thrombin activation of factor XIII and affects cross-linked fibrin structure. Blood 2000;96:988-95.

40. Balogh I, Szoke G, Karpati L, Wartiovaara U, Katona E, Komaromi I, et al. Val34Leu polymorphism of plasma factor 
XIII: biochemistry and epidemiology in familial thrombophilia. Blood 2000;96:2479-86.

41. Wartiovaara U, Mikkola H, Szoke G, Haramura G, Karpati L, Balogh I, et al. Effect of Val34Leu polymorphism on the activation of the coagulation factor XIII-A. Thromb Haemost 2000;84:595-600.

42. Kohler HP, Ariens RA, Whitaker P, Grant PJ. A common coding polymorphism in the FXIII A-subunit gene (FXIIIVal34Leu) affects cross-linking activity. Thromb Haemost 1998;80:704.

43. Raut S, Merton RE, Rigsby P, Muszbek L, Seitz R, Ariens RA, et al.Acollaborative study to establish the 1st International Standard for factor XIII plasma. J Thromb Haemost 2007;5:1923-9.

44. Dvilansky A, Britten AF, Loewy AG. Factor XIII assay by an isotope method. I. Factor XIII (transamidase) in plasma, serum, leucocytes, erythrocytes and platelets and evaluation of screening tests of clot solubility. Br J Haematol 1970;18:399-410.

45. Lorand L, Campbell-Wilkes LK, Cooperstein L. A filter paper assay for transamidating enzymes using radioactive amine substrates. Anal Biochem 1972;50:623-31.

46. Schmer G. A solid-phase radioassay for factor-XIII activity (fibrin stabilizing factor) in human plasma. Br J Haematol 1973;24:735-42.

47. Nelson JC, Lerner RG. Detection of factor XIIIa (active fibrinstabilizing factor) in normal plasma. Blood 1978;52:581-91.

48. Lorand L, Parameswaran KN, Velasco PT, Hsu LK, Siefring GE Jr. New colored and fluorescent amine substrates for activated fibrin stabilizing factor (Factor XIIIa) and for trans 4 has been
Anal Biochem 1983;131:419-25.

49. Nishida Y, Ikematsu S, Fukutake K, Fujimaki Memployed at the E. A new rapid and simple assay for factor XIII a dansylcadaverine incorporation and gel filtration. 1984;36:123-31.

50. Cooke RD, Holbrook JJ. Calcium and the assay plasma clotting factor XIII. Biochem J 1974;141:71-

51. Wenzel E, Christ G. The formation of ammonia plasma following recalcification. Thromb Diath 1969;22:575-6.

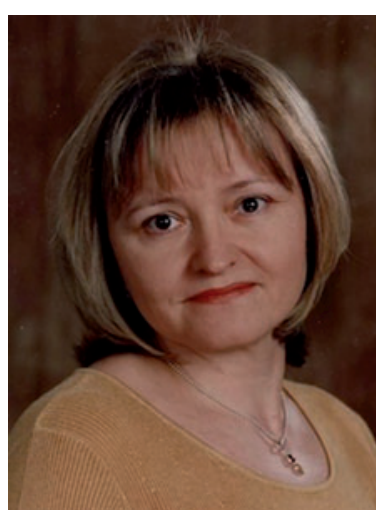

Éva Katona $\mathrm{PhD}$ is a senior research fellow at the Clinical Research Center of the Medical and Health Science Center, University of Debrecen. She became a certified clinical biochemist in 2006. Her main field of research is the development of new immunodiagnostic methods and reagents on the field of thrombosis and hemostasis. She is also involved in exploring the structure-function relationship of coagulation factors, especially factor XIII, and in studying the relationships between blood coagulation/fibrinolytic factors and vascular disorders. She has published 36 articles in scientific journals (total impact factor: 118). She has given lectures and practical courses for undergraduate and graduate students studying for a degree in clinical laboratory analytics and in clinical laboratory science for many years. She is involved in supervising PhD students and she is the secretary of a Doctoral School at the University.
52. Hellstern P, Schilz K, von Blohn G, Wenzel E. Determination of factor XIII activity and of factor XIII inhibitors using an ammonium-sensitive electrode. Thromb Haemost 1983;50:563-6.

53. Mousli S, Wakid NW. Ammonia production during clot retraction and its use in assay of fibrinoligase. Clin Chem 1977;23:1739-43.

54. Lawrie AS, Green L, Mackie IJ, Liesner R, Machin SJ, Peyvandi F. Factor XIII - an under diagnosed deficiency - are we using the right assays? J Thromb Haemost 2010;8:2478-82.

55. Schmidt ES, Schmidt FW. Glutamate dehydrogenase. In: Bergmayer HU, editor. Methods of enzymatic analysis Volume III. Enzymes 1: Oxidoreductases, transferases. Weinheim: Verlag Chemie GmbH, 1983:216-27.

56. Recommendations of the German Society for Clinical Chemistry: standardisation of methods for the estimation of enzyme activity in biological fluids. J Clin Chem Clin Biochem 1970;8:659-60.

57. da Fonseca-Wollheim F. Deamidation of glutamine by increased plasma gamma-glutamyltransferase is a source of rapid ammonia formation in blood and plasma specimens. Clin Chem 1990;36:1479-82.

58. Ajzner E, Muszbek L. Kinetic spectrophotometric factor XIII activity assays: the subtraction of plasma blank is not omissible [corrected]. J Thromb Haemost 2004;2:2075-7.

59. Meijer P. Clinically needed sensitivity for very low levels of Factor XIII: not yet proven for a new functional assay. Clin Chem Lab Med 2011;DOI:10.1515/CCLM.2011.649.

60. Kappel A, Stephan S, Christ G, Pechmann L, Duwe C, Fischer B,

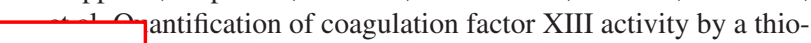
ased assay using factor XIII immuno-depleted plasma as for calibration. Clin Chem Lab Med 2010;48:1739-43. waran KN, Cheng XF, Chen EC, Velasco PT, Wilson JH, . Hydrolysis of gamma:epsilon isopeptides by cytosolic aminases and by coagulation factor XIIIa. J Biol Chem 2:10311-7.

, Hunfeld A, Specker E, Reiff C, Seitz R, Pasternack A highly sensitive fluorometric assay for determinauman coagulation factor XIII in plasma. Anal Biochem 2007;367:152-8.

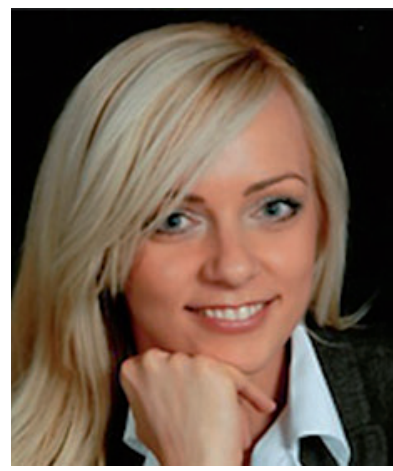

Krisztina Pénzes-Daku has a BSc in Medical Laboratory Diagnostics and an MSc in Molecular Biology. She has completed 3 years of $\mathrm{PhD}$ studies and is about to defend her thesis. She is employed as an assistant lecturer at the Clinical Research Center, University of Debrecen, Medical and Health Science Center. Her main area of research is the interaction of activated coagulation factor XIII with substrates, development of new factor XIII substrates and inhibitors. She has published scientific papers and had presentations at international scientific congresses. 


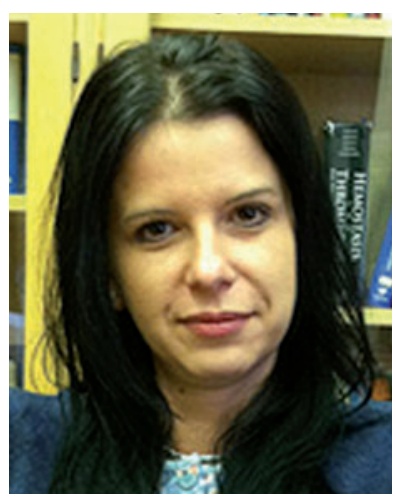

Éva Molnár has a BSc in Medical Laboratory Diagnostics. She is employed as a laboratory analyst at the Clinical Research Center, University of Debrecen, Medical and Health Science Center. She has been working on the development of new laboratory methods in the field of thrombosis and hemostasis, among others and on new factor XIII assays, and is highly experienced in the quality control of hemostatic diagnostic tests. She had several presentations at scientific congresses.

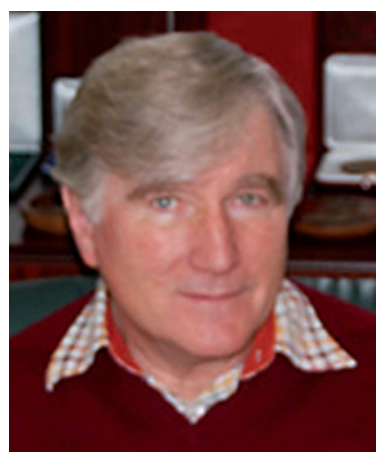

Prof. László Muszbek MD, $\mathrm{PhD}$ is a Member of the Hungarian Academy of Sciences. He has been employed at the University of Debrecen, Medical and Health Science Center and has been Chairman of the Department of Clinical Biochemistry and Molecular Pathology (1978-2004) and Director of Clinical Research Center (2004-present). He has held Visiting Fellowships and Professorships at the National Institute of Health, Bethesda, MD, University of Pennsylvania, Department of Pathology, Philadelphia, PA, Massachusetts General Hospital, Harvard University, Department of Pathology, Boston, MA. His main research areas include thrombosis and hemostasis and laboratory diagnostics. He has published 233 scientific papers, with an impact factor of 530 and been cited over 3000 times. He is involved in many international organizations including the Forum of European Societies of Clinical Chemistry as a Board Member and Treasurer (19931996), the International Federation of Clinical Chemistry and Laboratory Medicine as an executive board member (19971999), the European Thrombosis Research Organization as the General Secretary (2000-2003) and President (2007-2010), the International Society on Thrombosis and Haemostasis as a member of the Scientific and Standardization Committee (1997-2003) and has been a member of Senior Advisory Council (2003-present). He is an Editorial Board member of Blood Coagulation and Fibrinolysis, an Associate Editor of Thrombosis Research and is an Associate Editor of Thrombosis and Haemostasis (2007-2011). 